\title{
3D Sight Distance Calculation and Estimation of its Effect on Road Accidents in GIS Environment
}

\author{
Ali Sahaf ${ }_{(D},{ }^{1}$ Mostafa Mohammadi, ${ }^{2}$ and Ali Abdoli ${ }^{1}$ \\ ${ }^{1}$ Department of Civil Engineering, Ferdowsi University of Mashhad, Mashhad, Iran \\ ${ }^{2}$ Engineering Department, Civil Engineering Faculty, Science and Research Branch of Tehran, Isfahan, Iran \\ Correspondence should be addressed to Ali Sahaf; asahaf@um.ac.ir
}

Received 11 June 2021; Revised 2 July 2021; Accepted 2 August 2021; Published 12 October 2021

Academic Editor: S. Mahdi S. Kolbadi

Copyright (c) 2021 Ali Sahaf et al. This is an open access article distributed under the Creative Commons Attribution License, which permits unrestricted use, distribution, and reproduction in any medium, provided the original work is properly cited.

Nowadays, one of the main causes of human death is driving accidents. Across the world 1.2 million people die and several million people get injured annually as a result of road accidents. One of the main solutions that is important in resolving the problem of accidents is identifying the factors and their role and impact and the contribution of each in the accident. The stopping sight distance in the route is one of the initial factors in the driver's performance error as well as the occurrence of an accident, financial losses, and deaths. The geometric design of roads is generally designed according to two-dimensional rules and regulations. Hence, today, given the remarkable advances in computer science and programming, there are many possibilities for 3D modeling of the route. Therefore, the calculation of the stopping sight distance should be based on existing facts and new scientific achievements. On the contrary, the stopping sight distance is one of the factors affecting driving crashes; therefore, this paper tries to investigate the accident occurrence probability in other spots of the existing route by calculating 3D stopping sight distance and using drivers' free flow speed, as well as using the GIS software. In this way, the results of this study can pave the way for improving the geometric design of existing roads as well as prioritizing the correction of the accidental points of existing routes.

\section{Introduction}

Today, the statistics of accidents are very shocking and disturbing, and unfortunately, it can be described as a disaster. Hence, the facilitation and elimination of this dilemma is one of the main tasks of all. Considering the development of urbanization and the increased use of technology and machine life in the past decades, the current situation shows that, around the world, annual driving accidents cause 1.2 million deaths and injuries of more than 50 million people [1]. Studies by the World Health Organization (WHO) indicate that an increase of more than $65 \%$ of these figures has taken place over the past 20 years. Research indicates that $85 \%$ of these deaths and 90 of the annual disabilities are from low-income countries or middle-income countries and that deaths from driving accidents in the high-income countries will be reduced by up to $30 \%$ by 2020 , but in lower- and middle-income countries, it will continue to grow. In addition, in less developed countries, this is a more critical issue because of the lack of adequate oversight by agencies involved in accidents. Based on evaluations, more than $75 \%$ of road accidents occur in developing and in transit countries, while these countries account for only $32 \%$ of motor vehicles [2]. If the necessary measures are not taken to prevent accidents, driving accidents are predicted to reach the third level in 2020 from the ninth level of world illnesses and accidents in 1990 [3]. In Iran, about 23,000 people die annually and more than a thousand people are injured due to driving accidents [4]. Regardless of the wounded and the dead people in driving accidents, we are faced with such issues as medical expenses, financial losses, vehicle repair costs, ill mental effects on injured and their families, and loss or decrease in the efficiency and effectiveness of people involved in an accident [5].

Unfortunately, despite the implementation of preventive factors such as stricter regulations and restricting drivers by the police, as well as culture-building through the media, still 
an increase in the rate of accidents is observed in Iran [6]. Hence, more research is needed on accident intensification factors. Also, due to the increasing driving accidents and the complexity of the resulting issues, raising the level of general and specialized traffic awareness among the various strata of the population and traffic managers and the managers involved in this task has been necessary [7]. With regard to the above, it can be stated that crashes and road accidents are predictable and preventive so that many countries address major issues such as excessive speeding, seat belts, child safety devices, safety helmets, road design, and infrastructure and successfully make significant progress in reducing the number of accidents and reducing the frequency and severity of road traffic injuries [8]. One of the main reasons for the failure of many other countries to face this phenomenon is the inadequate understanding of this social problem as a multisectoral phenomenon with extensive and complex dimensions. This lack of knowledge causes many problems in correct orientation and adoption of basic solutions to the problem of accidents. One of these solutions, which is of great importance in the solution of the accident problem, is to identify the factors of the accident, their role and impact, and the contribution of each in the occurrence of an accident [9].

Among the various factors causing the accident, the sight distance of the driver's in different points of the road is of particular importance [10]. Sight in the driving lines in horizontal arches may be interfaced with side factors such as the trees and buildings or the slopes of the mountain, in case of which there is a need to cut the mountain. In the convex vertical arches, the line of sight may be covered by the arc. Also, night sight in concave vertical arches may be limited by vehicle light. Therefore, if these factors are neglected, they can provide the basis for the accident. On the contrary, the geometric design of the route is usually carried out according to the regulations of the two-dimensional space, while in practice (after the construction and operation of the route), the drivers control the vehicle in conditions influenced by slope, altitude, longitude, and latitude, in other words, based on the conditions in the three-dimensional space. The difference between the design and the operating conditions is more noticeable in parts of the rout with special geometric conditions, including the combination of horizontal and vertical arches. The sight distance in two-dimensional mode is plotted graphically on the plan and profile and recorded at successive intervals. The designer chooses the minimum distance recorded in the plan or profile [11]. Therefore, the results are conservative and do not express the exact extent of the available distance. Nowadays, due to the remarkable advances in computer science and programming, there are many possibilities for $3 \mathrm{D}$ and $4 \mathrm{D}$ modeling of the route [12]. Therefore, the sight distance design should be based on existing facts and new scientific achievements. As previously stated, analysis of the sight distance in routes is important for a driver in terms of safety, especially in maneuvers such as emergency stops or when the vehicle reaches the intersections. This analysis is important not only in the design phase of the road but also during road servicing.
Since the 1980s, the spatial information system (GIS) has been used in various transportation areas. The system has very powerful and updated software that has become popular among users in various fields [13]. One of the most popular and practical software is ArcGis, which has the necessary tools to calculate the sight distance in three dimensions from any point of the rout. Also, the use of the geographic information system in sight distance studies allows the use of information sources along the land, such as external barriers, such as trees and buildings, which reduce driver's sight. In this regard, in studies comparing sight distances obtained under two-dimensional and three-dimensional conditions and by conducting field surveys, the results obtained from geographic information system software were confirmed [14]. Therefore, considering the ability to calculate the three-dimensional sight distance, as well as the ability to detect obstacles such as trees and buildings around the route, the need for using geographic information system tools is deemed necessary to calculate sight distances. On the contrary, standards in different countries specify the minimum sight distance for maneuvering such as emergency stops, overtaking, or reaching an intersection, which indicates the importance of this issue. Also, three-dimensional analyzes based on actual conditions prevent the imposition of additional and unplanned costs for the projects to correct low-security points caused by ignoring actual conditions. Therefore, an essential requirement is felt for the basic and scientific identification of the factors involved in the incident, including the important issue of sight distance, which has a great potential for accident and is paid less attention.

1.1. Literature Review. Khatak and Shamayleh [15], using the topographic data obtained from the flight of a LIDARequipped aircraft, on a cross section of a two-way route, identified ten districts with problems in overtaking and stopping sight distance in the GIS environment and through field reviewing, all the problems were confirmed. Kim and Lovell [16] proposed a new 3D sight model that simultaneously took into consideration the horizontal and vertical factors, as well as other necessary factors such as road shoulder and external barriers. However, given the significant difference of the sight distance obtained from this method and other methods, it can be argued that this method has a considerably less cautious attitude in calculating sight distances. Merrow et al. used the finite element method to calculate the stopping sight distance in a combination of vertical-convex and horizontal arches on a twoway road. The results indicated that the ratio between the curved vertical arc parameter $(\mathrm{Kv})$ and the horizontal arc radius $(R)$ influenced the stopping sight distance. Castro et al. [17] compared the road sight distance analysis using other spatial information tools and other software and the results of the spatial information system tool were confirmed.

Barbel et al. at the University of Athens managed to design a software called $\mathrm{H} 11$ and presented it in an article as a model for calculating sight distances and three- 
dimensional evaluation of the route in separated and not separated routes. In another article, in 2013, entitled "Sight Distance," in order to analyze road safety using the spatial information system, Barbel et al. [18] introduced a tool to be installed in the ArcGIS software environment, which can be used to calculate three-dimensional sight distances. Similarly, in 2013, Maria Castro et al. introduced an enhancer tool for sight distance calculation for ArcGis software in the form of an article. The obvious feature of this enhancer is to calculate night sight distances and measure areas visible and invisible from any point. Also, according to the calculation of the available sight distance required from any point, a graphical comparison possibility is provided to the user. In 2016, Sahhaf and Abdoli, in their master's thesis, entitled 3D sight distance calculations and evaluation of its effect on free flow speed of drivers, obtained a mathematical formula to predict drivers' speed at any point of the route. In that study, the three-dimensional sight distance was calculated in the geographic information system, from BaghMalek to Izeh, Izeh to Dehdez, and BaghMalek to Seydoun routes in Khuzestan province using (TIN) vector modeling. Then, using the regression relations, the speed calculation formula was presented by tracking the speed of various drivers under conditions without road traffic.

\section{Materials and Methods}

2.1. Calculation of Sight Distance in GIS. Today, there are several software tools available for working with geospatial data, including advanced software such as the ArcGis developed by the ESRI Company. To calculate the sight distance between two points, software initially analyzes the natural land along the line of sight and then compares the elevation vector between the observer and the target and the height of the land along the line. If the natural land is plotted above the line of sight along the line of sight so that it disconnects the target's sight, then the target is considered invisible. On the contrary, the target is visible if the natural land is not above the line of sight. In this study, to calculate the stopping sight distance, the topographic files of the existing routes were modeled in Raster. The raster data consists of a regular and grid-like set of squares, of the same size and nondivisible cells, called pixels. Pixels are the smallest geometric component in the raster structure, and the position of each of the pixels is determined by its rows and columns. The number of pixels in the width and length of an image is called pixel dimension. The resolution of an image is determined by the number of pixels per inch. In this section, the Raster model of two existing roads is produced. In Figures 1 and 2, respectively, the raster models of the routes from Izeh to Dedez and BaghMalek to Izeh are shown.

At this stage, having all the information required and using the line-of-sight tool, the calculation of the existing 3D stopping sight distance is performed back and forth on both routes. This tool is capable of detecting the sight between the two points. For this purpose, by clicking on the Create Line of sight button a window opens in which both the Observer Offset and the Target Offset options are asked from the user.

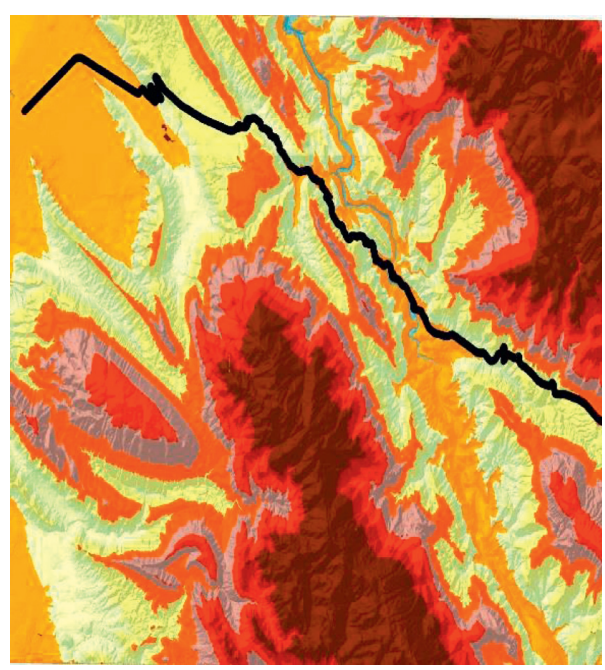

Figure 1: Raster model of the route of Izeh to Dedez.

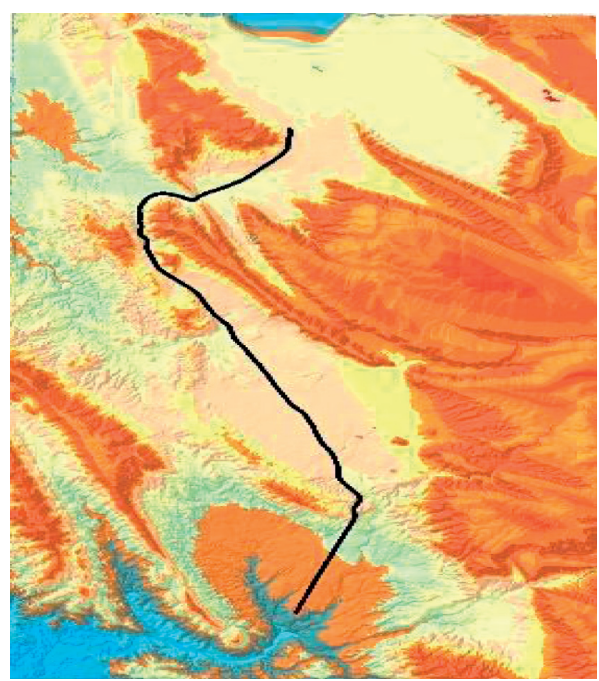

FIGURE 2: Raster model of the route of BaghMalek to Izeh.

The first option is related to the viewer's eye height, and according to the AASHTO, it is considered to be 1.08 and the second option is the height of the target or the barrier, which is considered to be 0.6 . By default, the software considers these options to be one and zero, respectively. After entering the regulation values the location of the viewer is determined by the first click and by the second click of the target point location is detected. By doing this, a virtual line with the colors of green and red appears between these two points. The green part of the line is a length of the route from which the target can be seen from the viewer's point, while this is not possible in the red part of this line. In Figures 3 and 4, respectively, the stopping sight distances obtained in the BaghMalek and Izeh routes are given.

2.2. Information Gathering and Modeling. This study requires topographic information of the area, crash rates, and speed of drivers at any point in the routes under the study. Therefore, topographic information of the area was provided 


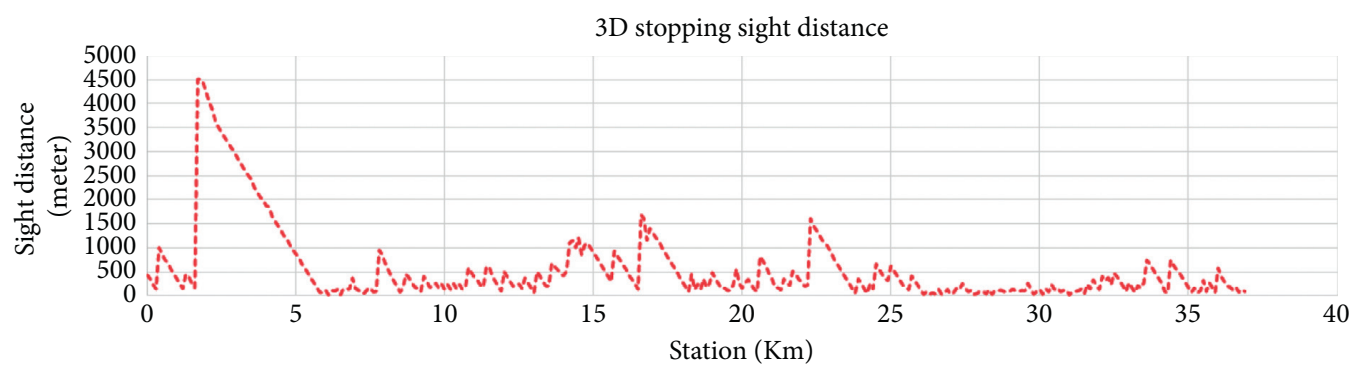

Figure 3: 3D stopping sight distance of the BaghMalek to Izeh route.

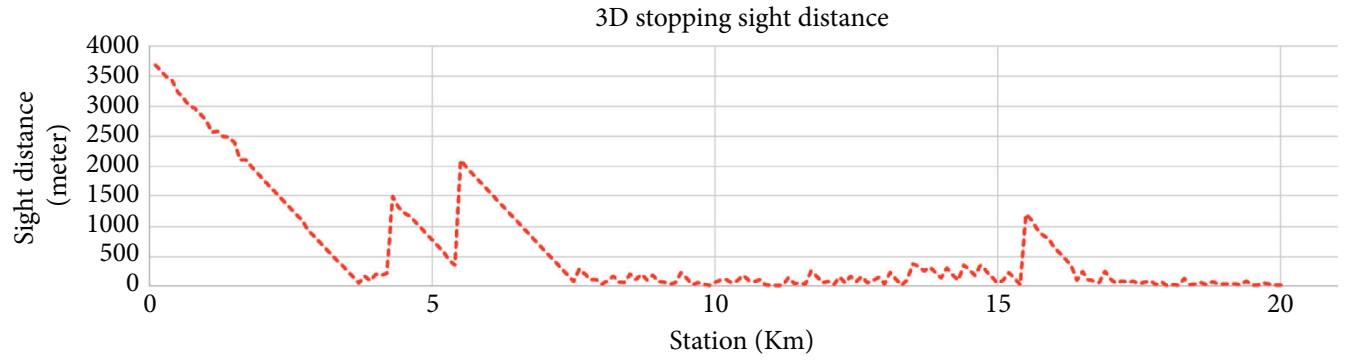

Figure 4: 3D stopping sight distance of the Izeh to Dehdez route.

by Sahhaf and colleague, from the Southwest region mapping organization, and the statistics of the route accidents was obtained by referring to the police of Khuzestan province, and speed of drivers was calculated according to

$$
S_{f f}=\left[\begin{array}{cc}
7821.86 \mathrm{ASD}^{0.002}-(7842)+\exp (-0.002535 * C C R+1.919 & 9 \leq \mathrm{ASD} \leq 1137.5 \\
97.5 & \mathrm{ASD}>1137.5
\end{array}\right]
$$

where $S_{f f}$ is free flow speed, ASD is available sight distance, and CCR is curvature change rate. Across the globe, crash statistics are recorded in various organizations so that they can be used if desired. In Iran, this information is also recorded in police stations in the provincial centers as well as in the provincial roads and urban planning departments of each province. After preparing the crash data in recent years in the routes studied in the Excel environment, the actual collection of information is completed. In order to examine the impact of several factors on each other, the weighted interpolation of effective factors is used. In this section, Kriging and co-Kriging tools are used for this interpolation. However, the difference between these two tools with other Arc GIS interpolation tools is that, firstly, according to the Raster data model, this tool is used for the more mountainous regions. Second, these tools are the most accurate interpolation tools, and third, it is possible to use more than two factors in interpolation in the co-Kriging tool. For this study, first, a raster file constructed in the software environment of the spatial information system was called; then, the accident points were located on the existing route (Figure 5).

According to the explanations and taking into account the total rate of crash data of the studied years, co-Kriging outputs from both BaghMalek to Izeh and Izeh to Dehdez have been presented. First, BaghMalek-Izeh route in Figure 6 and then the Izeh-Dehdez route in Figure 7 are determined, and the points whose crash data are available are located on the routes so that the software can use this information as well as the stopping sight distance and the free flow speed of the drivers to demonstrate a specific color scheme in its output.

In Figures 8 and 9, this color scheme is shown based on the software default, and the number of crashes is specified by a coefficient of 0 to 1 . The coefficient 1 presents the most probable occurrence of the accident and the zero number means the lowest probability of the accident; these numbers are placed next to the color scheme title output maps with regard to the minimum and maximum rates of the crashes.

2.3. Validation. In this research, we use a reverse method to verify this software so that, in one excel file that records the characteristics of the crash points, a specific point is eliminated to see whether software, according to the other points, puts this point in the same color scheme. As shown in Figure 10, there are 11 accident-prone points in the Izeh to Dehdez route, based on which the interpolation software 


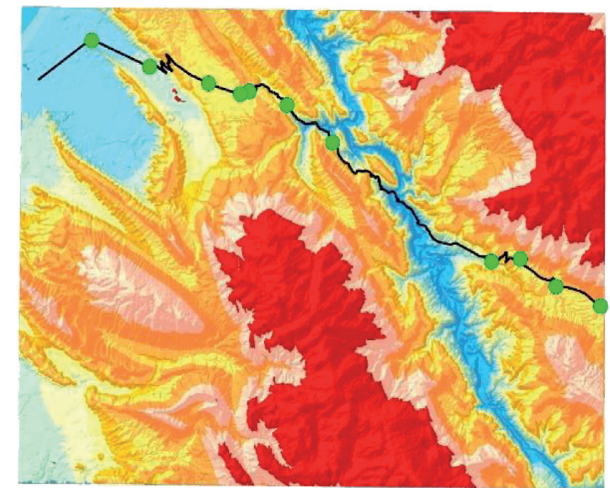

FIGURE 5: Location of accident points on the Izeh-Dedez route.

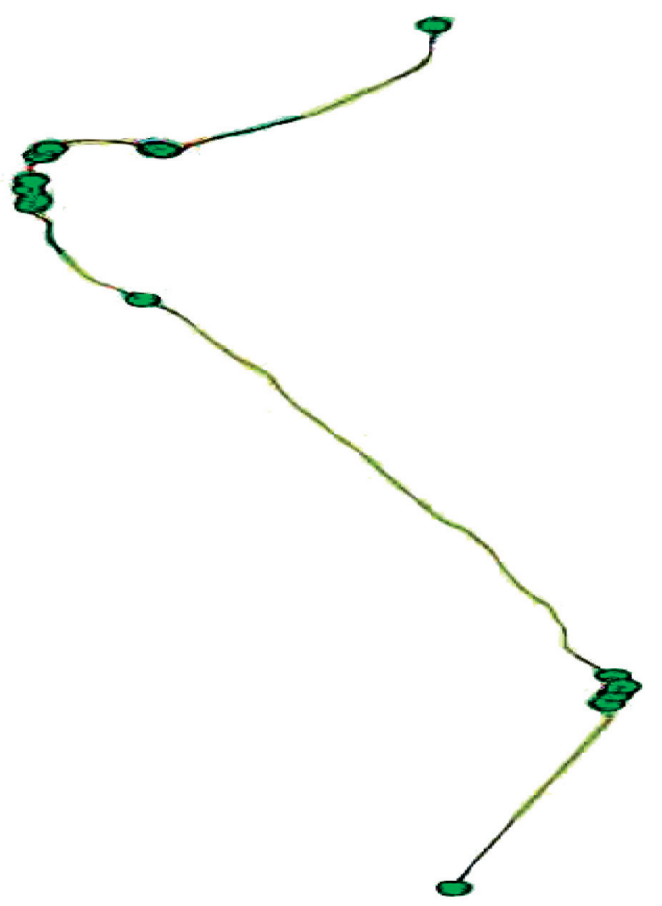

Figure 6: Co-Kriging modeling of BaghMalek to Izeh Route.

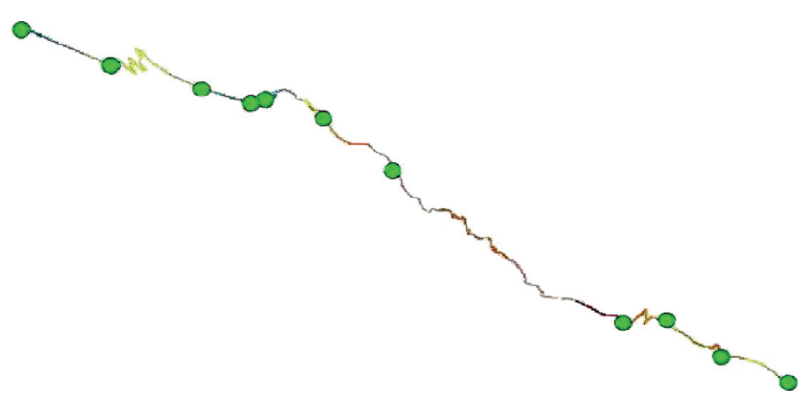

Figure 7: Co-Kriging modeling of the Izeh to Dehdez Route.

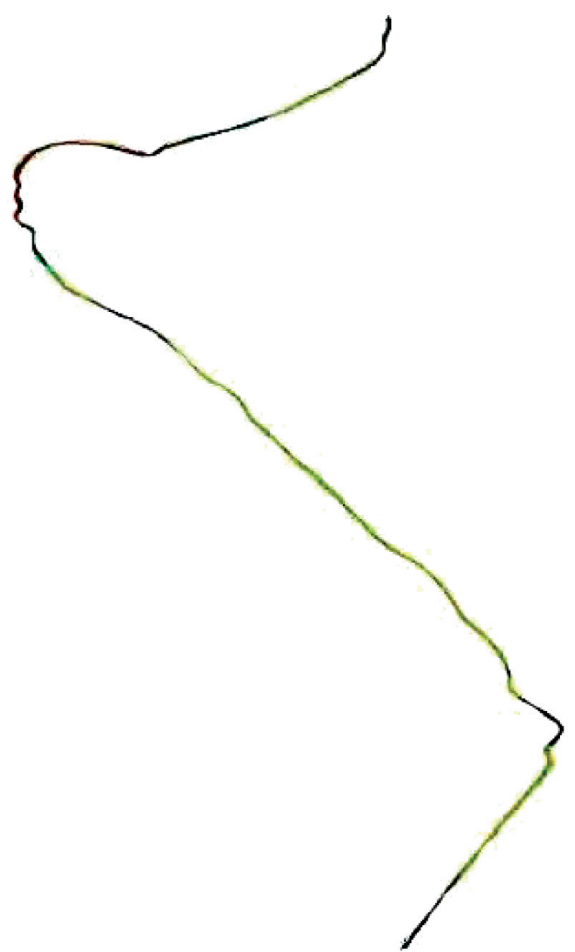

$0.052693151-0.199563755$

$0.199563755-0.297477491$

$0.297477491-0.361874011$

$0.351874011-0.384511923$

$0.384511923-0.417149836$

$0.417149835-0.460667051$

$0.460667051-0.506904093$

$0.506904093-0.55042131$

$0.55042131-0.621136786$

$0.621136788-0.746248782$

FIGURE 8: BaghMalek to Izeh route: estimation of the probability of an accident.

has performed its function. Now, the sixth point on the left side of the route was removed. It is in the yellow zone (Figure 11).

Now, with the implementation of co-Kriging interpolation with ten points on the Izeh-Dehdez route, according to the output shown in Figure 12, it is visible that the location of the sixth point is the border between the yellow and green spectrum, that is, approximately with more than $90 \%$ the yellow color spectrum, with an interval of less than 0.05 , has a probability coefficient difference, and is still in the same color spectrum. With this explanation, it can be said that the method of interpolation and estimation of this software is reliable more than ninety percent, and it can be deduced by having crash counts of several points of a route, as well as the stopping sight distance of the complete route and the drivers speed; a reasonable estimation of the probability of an accident can be predicted. 


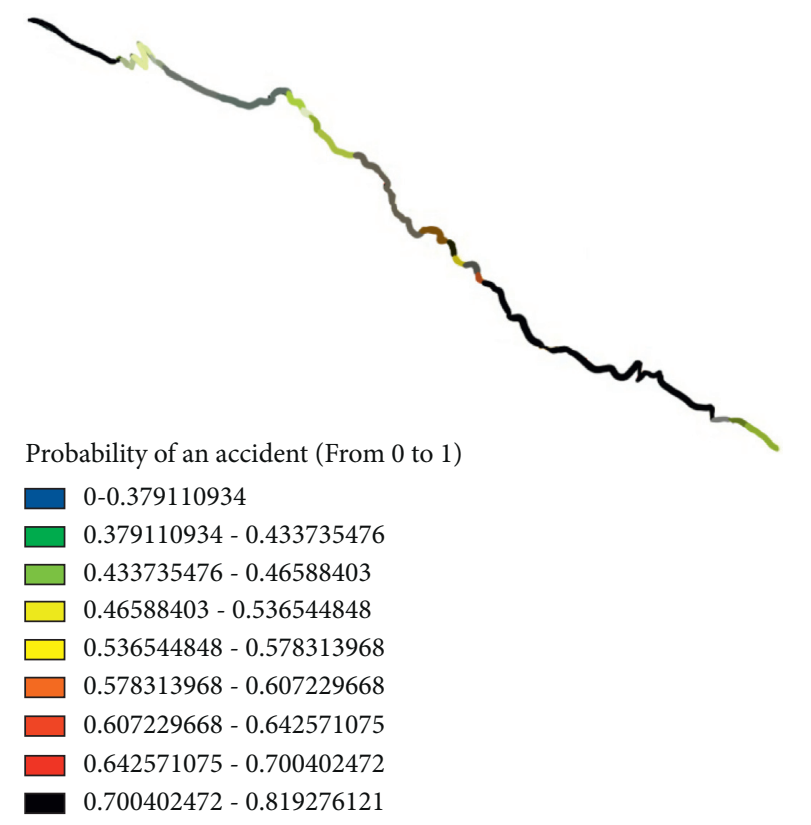

FIGURE 9: Izeh to Dehdez route: estimation of the probability of an accident.

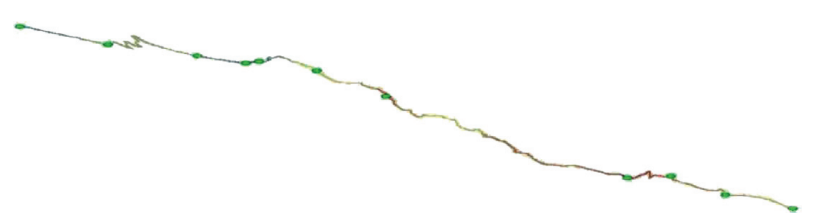

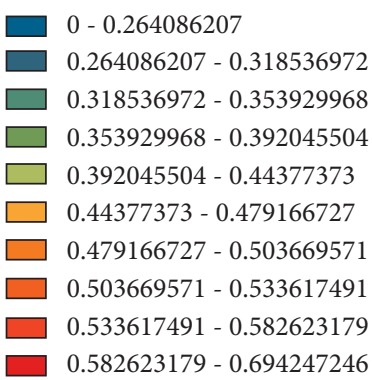

Figure 10: Accident points of the Izeh to Dehdez route.

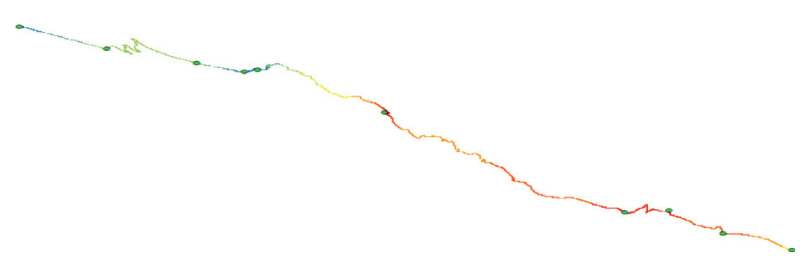

FIGURE 11: Removing the sixth point from the left side of the Izeh to Dehdez route.

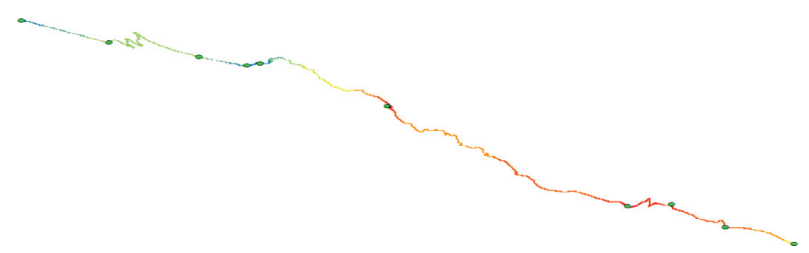

FIGURE 12: The result of the deletion of the sixth point of the Izeh to Dehdez route.

\section{Conclusion}

The goal of this research is to calculate the existing stopping sight distance by computer modeling of the two existing routes and then by using the speed of drivers and crash statistics, to determine the probability of occurrence of the accident at any point of the route. For this purpose, two mountainous routes were chosen. The selected routes include main roads of the northeast of Khuzestan province, and the connection route between Khuzestan province and other provinces, such as Chahar-mahal-and-Bakhtiari and Isfahan, which is also considered as one of the main routes connecting the south of the country to the north. To do this, it was necessary to provide various information, including the topography of the area, crash rates, and driver speeds at any point in the desired route. Therefore, topographic information of the area was provided by Sahhaf and colleague, from the Southwest region mapping organization, and the statistics of the route accidents was obtained by referring to the police of Khuzestan province and speed of drivers was calculated according to the formula. For the second part of this study, an Excel file was generated from multiple-point crash data in both routes over the course of several consecutive years. After the complete preparation of the system's required information, the co-Kriging tool was used to estimate the impact of crashes in other parts of the road.

After browsing the path file on the Raster Land model in the application, the crash points were added to it. Subsequently, the co-Kriging tool provided an output based on a different color scheme, which is characterized by coefficients that indicate the risk of accident along the route. With this analysis, as it was supposed, the sight distance and the crash rate have an inverse relationship. However, in this research, it was concluded that the $3 \mathrm{D}$ sight distance is far better and more accurate than the two-dimensional sight distance obtained from the existing regulations, and new software can be used to better serve the existing routes, and by analyzing and identifying points with a greater chance of accidents, a solution for better safety and reduction of road accidents can be obtained. Therefore, it is suggested to use this tool to design new routes and reduce the cost of further utilization of roads, as well as to compensate for the lack of available information and to reduce the cost of repair and 
maintenance. Meanwhile, the results prepared in this study can be used in other research studies to provide traffic safety routes, as well as in the preparation of more appropriate and more precise plans for correcting the accident points in the country [19-22].

\section{Data Availability}

The data used to support the findings of the study can be obtained from the corresponding author upon request.

\section{Conflicts of Interest}

The authors declare that there are no conflicts of interest regarding the publication of this paper.

\section{References}

[1] R. K. Mohammadi, M. Mirjalaly, M. Mirtaheri, and M. Nazeryan, "Comparison between uniform deformation method and genetic algorithm for optimizing mechanical properties of dampers," Earthquakes and Structures, vol. 14, no. 1, pp. 001-010, 2018.

[2] A. Ayati, F. Ghadiriyan, and M. Ahmadi, "Calculate the cost of damage to vehicles in road accidents in Iran," Transportation research journal, vol. 5th, no. 1, 2004.

[3] A. Zakaei Alamdari, H. Khodaveysi, and F. Falahi, "Conclusion on the relationship between economic development and road deaths in Iran: application of negative binomial distribution regression approach," Jornal of research, economic growth and development, vol. 2th, no. 5, pp. 184-205, 2011.

[4] E. Akyol, M. Alkan, A. Kaya, S. Tasdelen, and A. Aydin, "Environmental urbanization assessment using GIS and multicriteria decision analysis: a case study for Denizli (Turkey) municipal area," Advances in Civil Engineering, vol. 2018, Article ID 6915938, 7 pages, 2018.

[5] F. Bagheri and A. Sheykholeslami, "Analysis of research on the factors effecting the occurrence of accidents in suburban roads," Rahvar quarterly, vol. 8th, no. 15, pp. 93-116, 2011.

[6] L. R. Abdulrazzaq, M. N. Abdulkareem, M. R. Mat Yazid, M. N. Borhan, and M. S. Mahdi, "Traffic congestion: shift from private car to public transportation," Civil Engineering Journal, vol. 6, no. 8, pp. 1-12, 2020.

[7] F. Ahmadi, "Causes of accidents on urban roads," in Proceedings of the 7th Symposium on transportation and traffic, Tehran, Iran, August 2011.

[8] M. B. Ramiani and G. Shirazian, "Ranking and determining the factors affecting the road freight accidents model," Civil Engineering Journal, vol. 6, no. 5, pp. 1-18, 2020.

[9] A. Asghari Zamani, S. Moradi Monfared, SH. Zadvalikhaje, and Z. Ghasemi, "Investigation of spatial analysis of in-town crushes on human factors in it by using vicor model and GIS," in Proceedings of the 12th Symposium on transportation and traffic, Tehran, Iran, July 2013.

[10] T. E. Ahmed, K. M. Kheiralla, F. R. Awad Ahmed, R. A. Saeed, and $\mathrm{H}$. Alhumyani, "Design and implementation of multilayer GIS framework in natural resources management: red sea area," Complexity, vol. 2021, Article ID 5597707, 10 pages, 2021.

[11] A. Mansouriyan, H. Ghanavatian, and M. S. Monajem, "Three dimensional analyzed of stopping sight distance for combination horizontal curve with vertical curve simulated by
Spline function," in Proceedings of the 13th Symposium on transportation and traffic, Tehran, Iran, February 2014.

[12] D. B. Fambro, J. C. Collings, R. D. Vedova, J. P. Leisch, and J. M. Mason, Geometric Design: Past, Present and Future, Transportation Research Board, Washington, DC, US, 2008.

[13] S. Shadpoor, A. Pirouzi, H. Hamze, and D. Mazaheri, "Determination of Bodenstein number and axial dispersion of a triangular external loop airlift reactor," Chemical Engineering Research and Design, vol. 165, pp. 61-68, 2021.

[14] M. Castro, J. A. Anta, L. Iglesias, and J. A. Sánchez, "GIS-based system for sight distance analysis of highways," Journal of Computing in Civil Engineering, vol. 28, 2013.

[15] A. J. Khattak and H. Shamayleh, "Highway safety assessment through geographic information system-based data visualization," Journal of Computing in Civil Engineering, vol. 19, no. 4, pp. 407-411, 2005

[16] D. G. Kim and D. J. Lovell, "A procedure for 3-d sight distance evaluation using thin plate splines," in Proceedings of the 4th International Symposium on Highway Geometric Design, Valencia, Spain, June 2010.

[17] M. Castro, L. Iglesias, J. A. Sánchez, and L. Ambrosio, "Sight distance analysis of highways using GIS tools," Transportation Research Part C: Emerging Technologies, vol. 19, no. 6, pp. 997-1005, 2011.

[18] D. S. Barbel, M. CastroL, and I. Martínez, "Sight distance for road safety analysis using GIS," in Proceedings of the Esri Europe, Middle East and Africa User Conference, Munich, Germany, October 2013.

[19] A. T. M. Chou, V. Pérez, A. García, and M. A. R. Rojas, "Optimal 3D coordination to maximaise the available stopping sight distance in two-lane roads," in Proceedings of the 4th International Symposium on Highway Geometric Design, Valencia, Spain, June 2010.

[20] S. Fotis and M. Viviana, "Model for sight distance calculation and three-dimensional alignment evaluation in divided and undivided highways," in Proceedings of the 3rd International Conference on Road Safety and Simulation, Indianapolis, USA, September 2011.

[21] A. Sahhaf and A. Abdoli, "3D sight distance calculation and evaluation of its effect on free flow speed of drivers (case study: northeast connecting roads of Khouzestan-Iran)," International Ferdowsi University, Master Thesis ،faculty of the civil engineering, 2016.

[22] American Association of State Highway and Transportation Officials, A Policy on Geometric Design of Highways and Streets, American Association of State Highway and Transportation Officials, Washington, DC, USA, 2011. 